\title{
Test yourself-answer: multiple facial skin lesions associated with gingival hypertrophy in a pair of siblings
}

\author{
Nour Marroun $^{1}\left[\right.$ Diane Franck $^{1} \cdot$ Valérie Segers $^{1} \cdot$ Grammatina Boitsios $^{1}$
}

Received: 21 June 2021 / Revised: 20 August 2021 / Accepted: 27 August 2021 / Published online: 14 September 2021

(c) ISS 2021

The hand radiographs (Fig. 1) show multiple soft tissue tumefactions of the fingers tufts, with underlying osteolysis mainly on the distal phalanges (acrolysis), narrowing the distal interphalangeal joint without bone edge erosion. The head MRI shows multiple ovoid circumscribed subcutaneous masses characterized by a low signal on T1-weighted images and heterogeneous/high signal on T2-weighted images. They present with facilitated diffusion and intense and heterogeneous enhancement after contrast medium administration [1] (Fig. 2).

Skin nodules biopsies show micro-scattered cells with bland nuclei deposited in a densely hyalinized stroma (Fig. 3). The PAS test is positive with no significant cellular pleomorphism, mitoses, or necrosis [2].

Juvenile hyaline fibromatosis (JHF), first described in 1873 by Murray John [3], is a sporadic genetic disease with autosomal recessive transmission and a prevalence of 1/1.000.000 [4]; more than hundred cases are reported worldwide.

The disease is outlined by painful and progressive deposition of hyalinized fibrous material (collagen type VI) in the skin's extracellular matrix leading to the disease's clinical hallmark, the formation of subcutaneous nodules and enlarged gingiva [5]. The clinical features of JHF include joint disabilities (95.2\%), gingival hypertrophy (92.9\%),

The case presentation can be found at doi: https://doi.org/10.1007/ s00256-021-03898-2

Nour Marroun

nour_marroun@hotmail.com

Diane Franck

diane.franck@huderf.be

Valérie Segers

valerie.segers@chu-brugmann.be

Grammatina Boitsios

gboitsios@gmail.com

1 Hôpital Universitaire Des Enfants Reine Fabiola, Brussels, Belgium easy bleeding, and soft tissue nodules (85.7\%) most frequently seen in the cervicofacial region and osteolytic bone lesions (42.1-68.4\%) [6]. These findings are present in our siblings; they suffer from walking difficulties, recurrent joint pain, and joint deformation complicated by moderate to severe movement limitation mainly at the elbows and knees. Despite these limitations, they continue their daily activity (high school, university, and sports) with physiotherapy for pain control.

Hyaline fibromatosis syndrome (HFS) includes JHF (\#OMIM228600) and infantile systemic hyalinosis (ISH\#OMIM236490) that share many common features but with a different spectrum of severity. JHF tends to have a longer lifespan (until the fourth decade) and a better prognosis compared to the ISH which is the most severe form with the appearance of symptoms in the neonatal period.

JHF is caused by a mutation of the gene encoding for the capillary morphogenesis gene 2 protein (CMG2) located on chromosome 4q21 (OMIM228600). CMG2 encodes a membrane protein thought to be involved in the homeostasis of the extracellular matrix, the anthrax toxin receptor 2 (ANTXR2). To date, more than 15 different mutations are known for JHF [6].

There is no treatment for juvenile hyaline fibromatosis, and symptomatic treatment is often considered insufficient. The skin lesions are radio-resistant, and even after surgical resection, they often recur. Physiotherapy, intralesional steroids' injection, or systemic steroid therapy may be helpful in early joint disease [7].

Differential diagnoses are wide, including inherited gingival fibromatosis such as Zimmermann-Laband syndrome, where patients, in contrast to JHF, present hypoplasia and deformation of distal phalanges, joint hypermobility and mental retardation [8], and infantile myofibromatosis, characterized by multiple, symmetric lytic lesions in the long bone's metaphysis [9].

JHF is a rare, progressive, and debilitating connective tissue disease causing functional and severe esthetical 
Fig. 1 Left-hand and wrist radiographs of a the older brother at 18 years old and $\mathbf{b}$ the younger brother at 8 years showing skin nodules (arrows), acrolysis (asterisk), and narrowing of the distal interphalangeal joint without erosion of bone edges (circle)
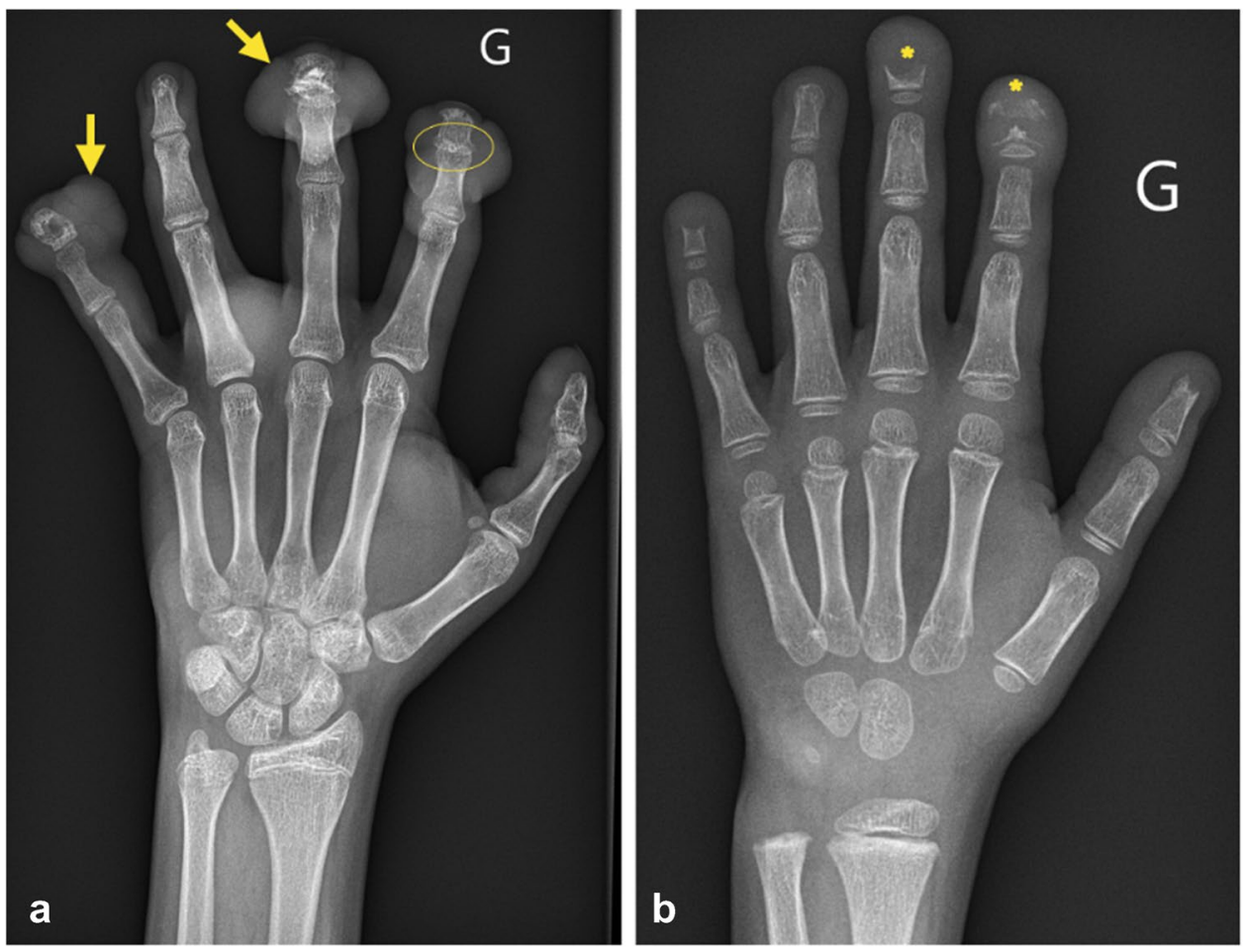
Fig. 2 Head and neck MRI of the younger brother at 8 years with T1-weighted images: a pre and $\mathbf{b}$ postcontrast illustrating the left subcutaneous lesion of the face (asterisk) with a heterogeneous and intense enhancement after intravenous contrast medium administration, c T2-weighted images with fat saturation showing heterogeneous high signal of the nodule, and $\mathbf{d}$ facilitated diffusion (high signal intensity) on the apparent diffusion coefficient map (ADCMAP)
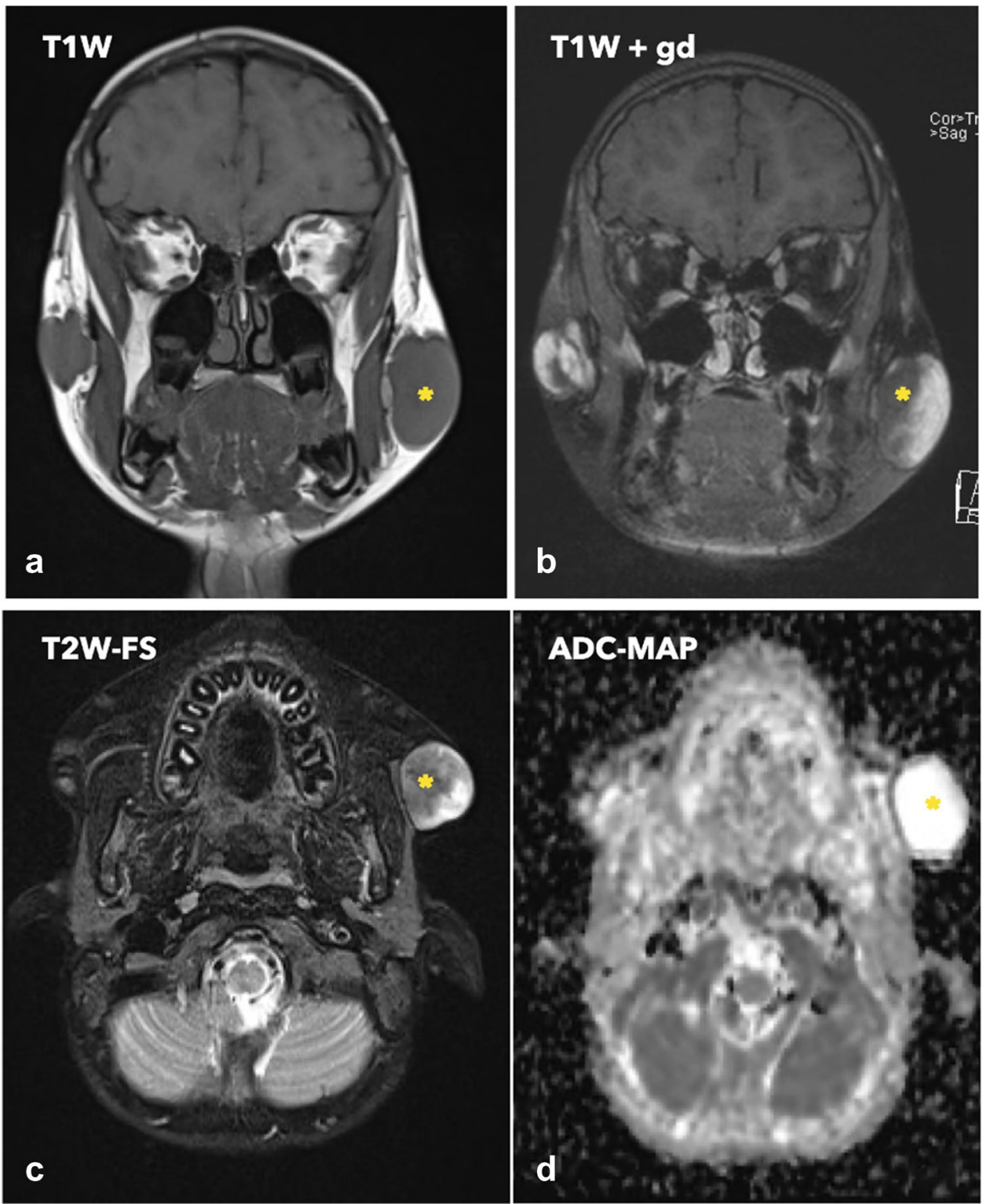


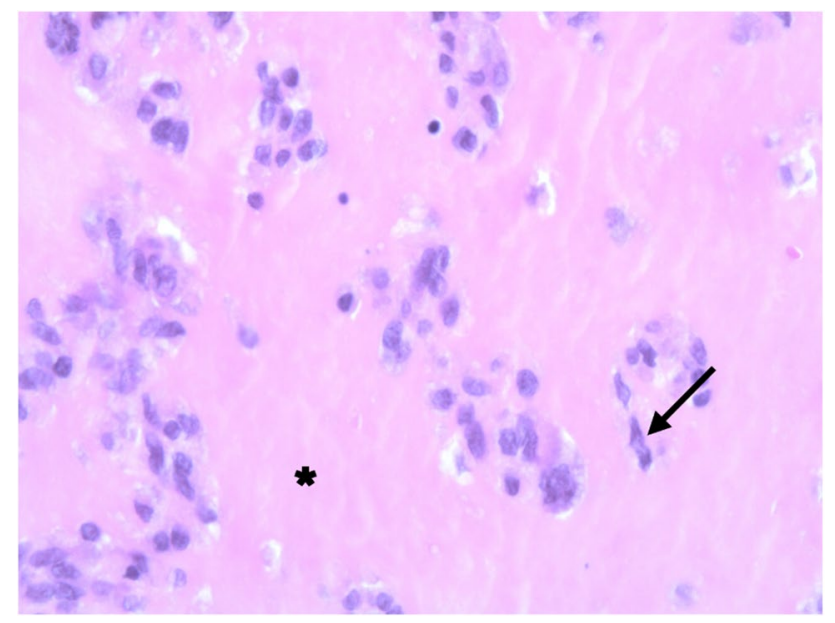

Fig. 3 Histopathological photomicrograph with hematoxylin and eosin staining of the chin lesion showing micro-scattered round and spindle (arrow) cells with bland nuclei deposited in a densely hyalinized stroma (asterisk) and positive PAS test

impairment without any definitive treatment. However, early conservative treatment can decrease the severity of the disease and improve the quality of life of these patients.

\section{Declarations}

Statement of informed consent The authors certify that they have obtained all appropriate patient consent forms. In the form, the patient(s) has/have given his/her/their consent for his/her/their images and other clinical information to be reported in the journal. The patients understand that their names and initials will not be published and due efforts will be made to conceal their identity, but anonymity cannot be guaranteed.

Conflict of interest The authors declare no competing interests.

\section{References}

1. Slimani S, Haddouche A, Haid S, Ladjouze-Rezig A. Juvenile hyaline fibromatosis: focus on radiographic features in adulthood. Rheumatol Int. 2011;31:273-6.

2. Mantri MD, Pradeep MM, Kalpesh PO, Pranavsinh RJ. Hyaline fibromatosis syndrome: a rare inherited disorder. Indian J Dermatol. 2016;61:580.

3. Murray J. On three peculiar cases of Molluscum Fibrosum in Children in which one or more of the following conditions were observed: hypertrophy of the gums, enlargement of the ends of the fingers and toes, numerous connective-tissue tumours on the scalp. Medico-Chir Trans. 1873;56:235-254.1.

4. Gawron K, Łazarz-Bartyzel K, Potempa J, Chomyszyn-Gajewska M. Gingival fibromatosis: clinical, molecular and therapeutic issues. Orphanet J Rare Dis. 2016;11:9.

5. Denadai R, Raposo-Amaral CE, Bertola D, Kim C, Alonso N, Hart T, et al. Identification of 2 novel ANTXR2 mutations in patients with hyaline fibromatosis syndrome and proposal of a modified grading system. Am J Med Genet A. 2012;158A:732-42.

6. Braizat O, Badran S, Hammouda A. Juvenile hyaline fibromatosis: literature review and a case treated with surgical excision and corticosteroid. Cureus [Internet]. 2020 [cited 2021 Mar 24]; Available from: https://www.cureus.com/articles/40500-juven ilehyaline-fibromatosis-literature-review-and-a-case-treated-withsurgical-excision-and-corticosteroid. Accessed 6 Oct 2020

7. Karande V, Andrade NN. Juvenile hyaline fibromatosis. Contemp Clin. Dent. 2018;9:484-7.

8. He L, Ping F-Y. Gingival fibromatosis with multiple unusual findings: report of a rare case. Int J Oral Sci. 2012;4:221-5.

9. Robbin MR, Murphey MD, Temple HT, Kransdorf MJ, Choi JJ. Imaging of musculoskeletal fibromatosis. Radiographics. 2001;21:585-600.

Publisher's note Springer Nature remains neutral with regard to jurisdictional claims in published maps and institutional affiliations. 